\title{
Abfraction Myth or Reality
}

\author{
Dr Pragya Tripathi, Dr Deepak Chopra, Dr Sukhchain Bagga \\ Sr Lecturer Dept of Periodontics, Inderprastha Dental College, Sahibabad, Ghaziabad (UP) India. \\ Reader Dept of Periodontics, Inderprastha Dental College, Sahibabad, Ghaziabad (UP) India. \\ Reader Dept of Periodontics, Inderprastha Dental College, Sahibabad, Ghaziabad (UP) India.
}

\begin{abstract}
Abfraction is the loss of tooth structure at the cervical region from heavy occlusal forces. It is described as one of the causes of lesions found along the cervical margins of teeth. This article critically reviews the literature in favour and against the theory of abfraction. From the literature there is little direct evidence supporting the theory of abfraction, apart from laboratory studies, to indicate that abfraction exists other than as a hypothetical component of cervical wear.
\end{abstract}

\section{Introduction}

Abfraction is the micro structural loss of tooth substance in areas of stress concentration. This occurs most commonly in the cervical region of teeth, where flexure may lead to a breaking away of the thin layer of enamel rods, as well as micro fracture of cementum and dentin ${ }^{1}$.

Since the publication of one of the text books for dentistry by anatomist and physiologist John Hunter in 1778, the definitions and classifications of the terms "attrition", "abrasion" and "erosion" have been in a state of confusion. Furthermore, the more recent introduction of the terms "abfraction" to designate stress induced non carious lesions have not resolved this dilemma fully ${ }^{2}$. During the two centuries that have ensured since Hunter published his work, a succession of investigators have unsuccessfully approached the problem of tooth surface denudation or wasting, using terms and conditions that were mutually contradictory ${ }^{3}$.

Up until now, research into the causes of abfractions seems to be divided into two camps- those who argue for tooth brushes and other artificial forces as the cause and those researchers who point to internal physiological sources as the culprit. The latter argument though not providing a complete explanation, does offer a significant clue to the real cause of this troubling phenomenon. In this review I have tried to solve the mystery behind abfraction $^{4}$.

\section{HISTORICAL BACKGROUND}

Powers et al in 1973 reported that enamel is weak in tension 5 .

Mc Coy in 1982 proposed that bruxism may be the primary cause of angled notches at the cementoenamel junction. The author postulated that tooth flexure from tensile stress led to cervical tooth breakdown ${ }^{6}$.

Lee and Eakle (1982) proposed a multi factorial etiology behind cervical tooth lesions, with a combination of occlusal stress, abrasion and erosion ${ }^{1}$.

Later, Lee and Eakle (1984) hypothesized that the primary etiological factor in wedge shaped cervical erosions was the impact of tensile stress from mastication and malocclusion. The wear is suggested to be created by a combination of bending and barreling deformations that cause alternating tensile and compressive stresses, which lead to a weakening of the enamel and dentin. The cyclic tension and compression may reach a fatigue limit and result in cracking or breakage of the tooth structure ${ }^{1}$.

Grippo in 1991 coined the term "abfraction" and defined it as the pathological loss of tooth substance caused by biomechanical loading forces that result in flexure and failure of enamel and dentin at a location away from the loading. Grippo suggested abfraction is the basic cause of all non carious cervical lesions ${ }^{2}$.

Levitch et al in 1994 proposed that the tensile phase of deformation may cause disruption, allowing water and other small molecules to penetrate between the prisms and prevent reestablishment of interprismatic bonds on release of the stress ${ }^{7}$.

Spranger(1995) in a review of literature, supported the multifactorial nature of the cervical lesions . The author suggested that the wear was related to the anatomy, the distribution of forces calculated from elastic deformation studies, development of caries, and occlusion and parafunction ${ }^{8}$.

Lee and Eakle (1996) suggested in a review, that the cervical fulcrum area of a tooth might be subjected to unique stress, torque and moments resulting from occlusal function, bruxing and parafunctional activity. These flexural forces would then disrupt the normally ordered crystalline structure of the thin enamel and underlying dentine by cyclic fatigue, leading to cracks, chips and rupture. Ultimately, the enamel breaks away at the cervical margin and progressively exposes the dentin, in which the process continues ${ }^{9}$. 
Engineering studies (Hammadeh and Rees, 2001; Lee et al., 2002; Rees et al., 2003) have demonstrated that when teeth are loaded in a horizontal direction, the effect of the stress becomes concentrated in the cervical region, causing flexure. ${ }^{10,11}$

\section{THE EVIDENCE SUPPORTING THE THEORY OF ABFRACTION:}

The theory of abfraction is based primarily on engineering analyses that demonstrate theoretical stress concentration at the cervical areas of tooth. While some recent stress studies support earlier findings, others have provided significant deviating information.

1. Much of the evidence for abfraction has been derived from finite element studies (Rees, 1998; Piotrowski et al., 2001; Rees etal.,2003; Geramy and Sharafoddin, 2003; Rees and Hammadeh, 2004). These computerized numerical procedures use discrete triangular elements, joined together to form nodes. The elements are ascribed certain physical properties, and a series of theoretical applied loads produces complex algebraic equations that are used to assess the impact of forces applied along the length of the model. Rees and coauthors used this concept to estimate the effect of repeated loading on restoration of cervical cavities. The hypothesis suggests that continued occlusal loading produced displacements and stresses under the buccal cervical enamel and dentin, increasing crack initiation and encouraging loss of the restoration 10,11, 12, 13, 14 .

2. Palamara and colleagues used scanning electron microscopy and profilometry to assess enamel loss in the cervical area of extracted teeth under cyclic occlusal loading, within the range of loads encountered in normal function. A tooth submerged in water showed enamel fractures at the CEJ, after only 200,000 cycles, equivalent to about 2.5 months of normal chewing. This increased after 500,000 cycles. One small area of enamel was completely chipped away from the $\mathrm{CEJ}^{15}$.

3. Hanaoka and colleagues in a similar study reported development of a crack network about $1.2 \mathrm{~mm}$ in width on the superficial cementum surface along the CEJ, which increased as the number of cycles increased. They stated that "mechanical microcracks on cementum and dentin may act as the initial contributor to the formation of cervical defects. Abfraction has a possibility of being the initial factor and the dominant progressive modifying factor in producing cervical lesions ${ }^{3}$.

4. Kuroe and colleagues in their studies indicated a positive correlation of cervical tooth surface lesions with tooth stability and periodontal support. They noticed that mobile teeth are less likely to develop the stress concentration that can produce abfraction ${ }^{16}$.

5. Staninec et al.(2005), in a laboratory study on thin sections of enamel and dentin taken from the cervical area of extracted unworn teeth, showed increased loss of mineralized tissue after stress and immersion in acid. The combination of erosion and stress upon the sections increased wear evenly over the length to a greater extent than when stress was applied singularly ${ }^{17}$.

6. Khan et al. (1992) in one paper reported the results of a study on 122 subjects with cervical wear. Cervical wear was compared with the appearance of the occlusal surface on the same tooth, and within the same subject. The study showed increased frequency of attrition on the occlusal surfaces in those teeth with cervical wear ${ }^{18}$.

7. Nohl and Setchell,(2000) in a clinical case study used strain gauges in three subjects. In the healthy subjects, increased strain developed along the cervical areas, but there was no information available to compare the results with those from controls 19 .

8. Xhonga (1977) tested the hypothesis that occlusion has some influence on cervical wear. In this study on 30 subjects the author reported a significant association between the presence of occlusal and cervical wear. Increased incidence of cervical wear in bruxists as compared with non-bruxists was reported ${ }^{4}$.

\section{THE EVIDENCES AGAINST THE THEORY OF ABFRACTION}

The theory of abfraction is based primarily on engineering analyses that demonstrate theoretical stress concentration at the cervical areas of teeth. While there are a number of studies linking occlusal forces to tooth flexure few controlled studies exist that demonstrate the relationship between occlusal loading and abfraction lesions ${ }^{20}$.

1. Most of the damage of this nature is to the buccal surfaces. Contradictory evidence from the finite element studies suggests that the lingual walls of teeth should be equally as susceptible to cervical wear as are the buccal walls(Rees,2002) but this is not reported by the clinical findings, where lingual lesions are comparatively rare (Radentz et al., 1976). The lesions tend to be much worse on the buccal surfaces of the premolars and canines where patients are likely to place the most brushing force. It becomes progressively worse as one proceeds from the posterior teeth to the anteriors . Furthermore the most affected teeth tend to be in buccal version. The teeth in which linguals are affected are mostly found mesial to an edentulous space ${ }^{21}$.

2. There is little or no evidence of these lesions in prehistoric skulls, even though the teeth show considerable occlusal wear from mulling tough and fibrous foods. All the cervical erosions found in historic skulls seem to begin after the invention of tooth powders and tooth brushes in the $16^{\text {th }}$ century ${ }^{22}$. 
3. The damage seems to stop at the gingival crest instead of at the crest of the bone, which is where the theory of abfraction suggests the flexure should be the worst ${ }^{3}$.

4. Not all persons with cervical lesions demonstrate occlusal wear, which would indicate a bruxing habit, and not all persons with severe bruxing occlusal wear exhibit cervical non carious lesions ${ }^{4}$.

5. Frequently the teeth in which there is ongoing erosion of buccal tooth structure have no opponent in the opposite arch. It can be shown that the damage is ongoing, or that the damage began after the extraction of the opposing tooth, then bruxing cannot be a factor in producing it ${ }^{3}$.

\section{EXPLANATION REGARDING EVIDENCES AGAINST THE THEORY OF ABFRACTION}

1. These lesions are limited almost exclusively to the buccal and labial cervical areas of teeth. This finding is explained by a synergistic role of abrasion and abfraction. Abrasion-abfraction is the loss of tooth substance caused by friction from an external material on an area in which stress concentration due to loading forces may cause tooth substance to break away. Such a synergistic tooth destructive effect may be observed cervically when toothbrushing abrasion exacerbates abfraction to produce wedge shaped lesions ${ }^{3}$.

2. Abfractions are not found in prehistoric times but are quite common in today's contemporary society. This observation is due to lifestyle differences between the two time periods. These lifestyle differences include breastfeeding attitudes, bottle-feeding and pacifier use in contemporary times. In prehistoric time women had no choice other than to breastfeed their newborns. Breastfeeding reduces the risk of both malocclusions and tongue thrusting. The longer an infant is breastfed the better the chance the infant will develop a proper swallowing pattern. The swallowing action influences the position of the teeth in the dental arch and the height of the palate. Strong lateral forces generated by tongue thrusting may result in the development of abfractive lesions ${ }^{22}$.

3. Combined abrasion-abfraction effect also explains why the damage seems to stop at the gingival crest instead of at the crest of the bone ${ }^{3}$.

4. Abfraction on teeth that do not have wear facets may be due to the traumatic lateral forces generated by tongue thrust. Teeth that have wear facets but do not have abfractions simply have responded differently to that force. The response depends on the local environment, including the health of the individual and surrounding periodontium ${ }^{22}$.

5. Teeth that are out of occlusion could develop cervical lesions, this finding can be explained by the combined effect of abrasion and corrosion. This could occur from the frictional effects of a toothbrush on the superficially softened surface of a tooth. Teeth that are out of occlusion frequently extrude, thus exposing the vulnerable dentin ${ }^{3}$.

6. GCF is acidic in nature and thus the occasional finding of subgingival cervical lesions may well be examples of corrosion-abfraction process ${ }^{3}$.

\section{Conclusion:}

Nearly all the research on the relationship of occlusal forces to cervical lesions shows that teeth do, indeed flex in the cervical region under bruxing loads, but none seems to cite actual damage caused by this phenomenon without an abrasive or erosive component applied as well, so the role of occlusal loading in noncarious cervical lesions appears to be a part of a multifactorial event that may not necessarily follow the proposed classic abfraction mechanism. Till date theory of abfraction is not completely understood, more research particularly clinical is needed to establish the validity of abfraction existing as an entity.

\footnotetext{
References

[1]. Lee WC, Eakle WS. Possible role of tensile stress in the etiology of cervical erosive lesions of teeth. J Prosthetic Dent 1984;52(3):374-80.

[2]. Grippo JO. Abfractions: a new classification of hard tissue lesions of teeth. J Esthet Dent 1991; 3(1):14-9.

[3]. Grippo JO, Simring M, SchreinerS. Attrition, abrasion, corrosion and abfraction revisited. JADA 2004; 135(8):1109-18

[4]. Bartlett D.W, Shah P. A critical review of non carious cervical (wear) lesions and the role of abfraction, erosion and abrasion. J Dent Res 2006; 85(4):306-12.

[5]. Powers JM, CraigRG, Ludema KC. Frictional behavior and surface failure of human enamel. J Dent Res1973; 52:1327-31.

[6]. Mc Coy G. The etiology of gingival erosion. J Oral Implantol. !982; 10: 361-62.

[7]. Levitch LC, Bader JD, Shugars DA, Heymann HO. Non-carious cervical lesions. J Dent Res 1994; 22 : 195-207.

[8]. Spranger H. Investigation into the genesis of angular lesions at the cervical region of teeth. Quintessence Int 1995; 26: 149-54

[9]. Lee WC, Eakle WS. Stress-induced cervical lesions: review of advances in the past 10 years. J Prosthetic Dent 1996; 75: $487-94$.

[10]. Rees JS, Hammadeh M. Undermining of enamel as a mechanism of abfraction lesion formation: a finite element study. Eur J Oral Sci 2004; 112: 347-52.

[11]. Lee et al. Stresses at the cervical lesion of maxillary premolar- a finite element investigation. J Dent 2002; 30: 283-90.

[12]. Rees JS. The role of cuspal flexure in the development of abfraction lesions: a finite element study. Eur J Oral Sci 1998; 106: 1028-32.

[13]. Piotrowski BT et al. Examining the prevalence and characteristics of abfraction like cervical lesions in a population of US veterans. JADA 2001; 132: 1694-1701.
} 
[14]. Geramy A, Sharafoddin F. Abfraction: 3D analysis by means of the finite element method. Quintessence Int 2003; 34: 526-33.

[15]. Palamara D, Palamara JE, Tyas MJ, Pintado M, Messer HH. Effect of stress on acid dissolution of enamel. Dent Mater 2001; 17(2): 109-15.

[16]. Kuroe T, Itoh H, Caputo AA, Kunuma M. Biomechanics of cervical tooth structure lesions and their restoration. Quientessence Int 2000; 31: 267-74.

[17]. Staninec $\mathrm{M}$ et al. Dentin erosion stimulation by cantilever beam fatigue and $\mathrm{pH}$ change. J Dent Res 2005; 84: 371-75.

[18]. Khan F, Young WG, Shahabi S, Daley TJ. Dental cervical lesions associated with occlusal erosion and attrision. Aust Dent J 1999; 44: $176-86$

[19]. Nohl FS, Setchell DJ. Surface strains induced by measured loads on teeth in vivo: a methodological study. Eur J Prosthodont Rest Dent 2000; 8: 27-31.

[20]. Litonjua LA, Andreana S, Bush PJ, Cohen RE. Noncarious cervical lesions and abfractions 2003; 134(7): 845-50.

[21]. Radentz WH, Barnes GP, Cutright DE. A survey of factors possibly associated with cervical abrasion of tooth surfaces. J Periodontol 1976; 47: 148-54.

[22]. Palmer B. Abfractions. JADA 2006; 137(4):438-39. 